\title{
Optimal Spline Generators for Derivative Sampling
}

\author{
Shayan Aziznejad*, Alireza Naderi ${ }^{\dagger}$, and Michael Unser* \\ *Biomedical Imaging Group, EPFL, Lausanne, Switzerland \\ ${ }^{\dagger}$ Department of Electrical Engineering, Sharif University of Technology, Tehran, Iran \\ Emails: shayan.aziznejad@epfl.ch,naderi_alireza@ee.sharif.edu,michael.unser@epfl.ch
}

\begin{abstract}
The goal of derivative sampling is to reconstruct a signal from the samples of the function and of its first-order derivative. In this paper, we consider this problem over a shiftinvariant reconstruction subspace generated by two compactsupport functions. We assume that the reconstruction subspace reproduces polynomials up to a certain degree. We then derive a lower bound on the sum of supports of its generators. Finally, we illustrate the tightness of our bound with some examples.
\end{abstract}

\section{INTRODUCTION}

The generalized sampling framework was introduced by Papoulis in [1] as an extension of Shannon's celebrated sampling theorem [2]. It aims at the reconstruction of a bandlimited signal from the samples of its response to a set of linear shift-invariant systems [3]-[8]. An important special case of Papoulis's framework is derivative sampling, where the linear systems are assumed to be differentiators of certain degrees [3], [9]-[12].

While the pioneering works on derivative sampling were focused on recovering band-limited functions [9], [10], Unser and Zerubia proposed a generalized sampling framework for signals without this constraint [13], [14]. Here, we consider their framework for derivative sampling by performing the reconstruction over a finitely generated shift-invariant subspace of $L_{2}(\mathbb{R})$. The structure of such subspaces has been investigated by de Boor et al. [15], [16]. This includes reconstruction over spline spaces and wavelets; these schemes have been shown to outperform classical methods [17]-[19].

We consider the problem of finding an approximation $\tilde{f} \in V$ of a function $f: \mathbb{R} \rightarrow \mathbb{R}$ given the regular samples $\{f(k)\}_{k \in \mathbb{Z}}$ and $\left\{f^{\prime}(k)\right\}_{k \in \mathbb{Z}}$ of the function and of its derivative. The approximated function $\tilde{f}$ lies in a shift-invariant subspace $V$ generated by two functions (minimum number of required generators). We ask that it be consistent with the given samples, so that

$$
\tilde{f}(k)=f(k), \quad \tilde{f}^{\prime}(k)=f^{\prime}(k), \quad \forall k \in \mathbb{Z} .
$$

The complexity of the reconstruction algorithm is in direct relation with the support size of the generators. Consequently, it is desirable to find generators of shortest support. The other important feature in this scheme is the approximation power of the reconstruction algorithm. This is known to be related to the polynomial reproducibility of the reconstruction subspace [16], [20].

In this paper, we show that the ability to reproduce polynomials come with a lower bound on the sum of supports of the generators of the reconstruction subspace. This bound determines a fundamental trade-off between the two desirable properties of the space.

The paper is organized as follows: In Section II, we formulate the problem and explore the reconstruction algorithm. We then propose and prove our main result in Section III. Finally, in Section IV, we discuss our theorem and provide some examples.

\section{Problem Formulation}

The subspace $V \subseteq L_{2}(\mathbb{R})$ is said to be (integer) shiftinvariant if, for any $f \in V$ and any integer shift value $k \in \mathbb{Z}$, $f(\cdot-k) \in V$. For a funtion $\phi \in L_{2}(\mathbb{R})$, the space generated by $\phi$ is denoted by $S(\phi)$ and is defined as [15]

$$
S(\phi)=\overline{\operatorname{Span}}\left(\{\phi(\cdot-k)\}_{k \in \mathbb{Z}}\right) \subseteq L_{2}(\mathbb{R}) .
$$

Similarly, the space generated by the finite collection of functions $\phi_{1}, \phi_{2}, \ldots, \phi_{N} \in L_{2}(\mathbb{R})$ is denoted by $S\left(\phi_{1}, \phi_{2}, \ldots, \phi_{N}\right)$ and is defined as

$$
S\left(\phi_{1}, \phi_{2}, \ldots, \phi_{N}\right)=S\left(\phi_{1}\right)+S\left(\phi_{2}\right)+\cdots+S\left(\phi_{N}\right) .
$$

Throughout this paper, we assume the joint Riesz condition for the generator functions $\phi_{n} \in L_{2}(\mathbb{R})$ [21]. It requires the existence of positive constants $m, M \in \mathbb{R}^{+}$such that, for any collection of sequences $a_{n}[\cdot]=\left(a_{n}[k]\right)_{k \in \mathbb{Z}} \in \ell_{2}(\mathbb{Z})$ for $n=1,2, \ldots, N$, we have that

$$
m\|\vec{a}\|_{\ell_{2}} \leq\left\|\sum_{n=1}^{N} \sum_{k \in \mathbb{Z}} a_{n}[k] \phi_{n}(\cdot-k)\right\|_{L_{2}} \leq M\|\vec{a}\|_{\ell_{2}},
$$

where $\vec{a}[k]=\left(a_{1}[k], a_{2}[k], \ldots, a_{N}[k]\right)$ and $\|\vec{a}\|_{\ell_{2}}=$ $\left(\sum_{n=1}^{N}\left\|a_{n}\right\|_{\ell_{2}}^{2}\right)^{\frac{1}{2}}$. This condition ensures the unique and stable representation

$$
f(\cdot)=\sum_{n=1}^{N} \sum_{k \in \mathbb{Z}} a_{n}[k] \phi_{n}(\cdot-k), \quad \forall n: a_{n}[\cdot] \in \ell_{2}(\mathbb{Z})
$$

for any $f \in S\left(\phi_{1}, \phi_{2}, \ldots, \phi_{N}\right)$.

To ensure a one-to-one correspondence, we pick the reconstruction space $V=S\left(\phi_{1}, \phi_{2}\right)$ with the two generators $\phi_{1}, \phi_{2} \in L_{2}(\mathbb{R})$. In addition to the joint Riesz condition on $\phi_{1}$ and $\phi_{2}$, derivative sampling assumes at least some smoothness on the elements of the reconstruction subspace. To be precise, we assume that the $\phi_{i}$ are differentiable functions with continuous derivative (i.e., $\phi_{1}, \phi_{2} \in C^{1}(\mathbb{R})$ ). We also assume that the target function along with its derivative are $L_{2}$ functions. In other words, we assume that the target function 
lies in the Sobolev space of order 1 which is denoted as $W_{2}^{1}(\mathbb{R})[22]$.

The goal of the reconstruction algorithm is to find the coefficients $\left(a_{n}[k]\right)_{k \in \mathbb{Z}} \in \ell_{2}(\mathbb{Z})$ for $n=1,2$ such that, for all $\ell \in \mathbb{Z}$

$$
\begin{aligned}
& f(\ell)=\sum_{k \in \mathbb{Z}} a_{1}[k] \phi_{1}(\ell-k)+\sum_{k \in \mathbb{Z}} a_{2}[k] \phi_{2}(\ell-k), \\
& f^{\prime}(\ell)=\sum_{k \in \mathbb{Z}} a_{1}[k] \phi_{1}^{\prime}(\ell-k)+\sum_{k \in \mathbb{Z}} a_{2}[k] \phi_{2}^{\prime}(\ell-k) .
\end{aligned}
$$

By defining $\vec{f}[k]=\left(f(k), f^{\prime}(k)\right)$ and $\vec{\phi}[k]=\left(\phi_{i}[k], \phi_{i}^{\prime}[k]\right)$ for $k \in \mathbb{Z}$, we can rewrite (6) in vectorial form as

$$
\vec{f}[\ell]=a_{1} * \vec{\phi}_{1}[\ell]+a_{2} * \vec{\phi}_{2}[\ell], \quad \forall \ell \in \mathbb{Z},
$$

where the convolution operator applies component-wise. We denote the z-transform of the sequence $c[k]$ as $C(z)$. Then by taking the z-transform of (7), we obtain that

$$
\vec{F}(z)=A_{1}(z) \vec{\Phi}_{1}(z)+A_{2}(z) \vec{\Phi}_{2}(z) .
$$

A crucial assumption for derivative sampling is the invertibility of the matrix $\boldsymbol{\Phi}(z)=\left[\begin{array}{ll}\vec{\Phi}_{1}(z) & \vec{\Phi}_{2}(z)\end{array}\right]$ for all $z \in \mathbb{C}$ with $|z|=1$. The inverse matrix $\boldsymbol{\Phi}^{-1}(z)$ specifies a digital reconstruction filter $\vec{a}[k]=(\mathbf{H} * \vec{f})[k]$, where $\vec{a}[k]=\left(a_{1}[k], a_{2}[k]\right)$ and $\mathbf{H}=\left[h_{i, j}\right]$ is the inverse z-transform of $\boldsymbol{\Phi}^{-1}(z)$. This yields

$$
a_{i}[k]=h_{i, 1} * f[k]+h_{i, 2} * f^{\prime}[k]
$$

for $i=1,2$. The filter $\mathbf{H}$ is not necessarily FIR. However, it can be implemented efficiently using recursive methods [17]. Its computational complexity is also proportional to the support of $\phi_{1}, \phi_{2}$. Note that the coefficients $a_{i}[k]$ are uniquely determined from (9). Hence, the consistent-approximation problem has a unique solution in the space $S\left(\phi_{1}, \phi_{2}\right)$.

Ideally, we are looking for a scheme where $\mathbf{H}$ is the identity filter, which translates into generators being interpolatory. Specifically, a pair of generators $\vec{\phi}_{\text {int }}=\left(\phi_{\text {int }, 1}, \phi_{\text {int }, 2}\right)$ is said to be interpolatory if, they satisfy (7) with $\vec{a}[k]=\vec{f}[k]$ for all $k \in \mathbb{Z}$. To construct such generators, we simply need to apply the digital filter $\mathbf{H}^{T}$ (the inverse z-transform of $\boldsymbol{\Phi}^{-T}$ ) on the original generators $\vec{\phi}$, which results in

$$
\vec{\phi}_{\text {int }}(x)=\sum_{k \in \mathbb{Z}} \mathbf{H}^{T}[k] \vec{\phi}(x-k) .
$$

This procedure yields the unique intepolatory generators of the space. However, the resulting generators are not necessarily compactly supported.

\section{MAIN ResulT}

An important property of the reconstruction subspaces is their ability to reproduce polynomials.

Definition 1. We say that the space $V=S\left(\phi_{1}, \phi_{2}, \ldots, \phi_{N}\right)$ reproduces polynomials of degree up to $M$ if, for all $m=$ $0,1, \ldots, M$, there exist sequences of coefficients $\left(p_{n}^{(m)}[k]\right)_{k \in \mathbb{Z}}$ (not necessarily in $\ell_{2}(\mathbb{R})$ ) such that

$$
x^{m}=\sum_{n=1}^{N} \sum_{k \in \mathbb{Z}} p_{n}^{(m)}[k] \phi_{n}(x-k), \quad \forall x \in \mathbb{R} \backslash\{0\} .
$$

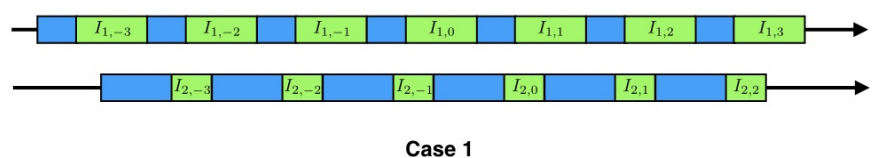

Case 1

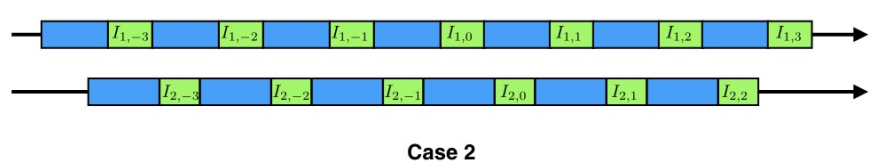

Fig. 1. Two possible cases for the intersection of $I_{1}$ and $I_{2}$. Case 1: an interval is completely contained in $I_{1, T_{1}}$ and $I_{2, T_{2}}$ for some $T_{1}$ and $T_{2}$. Case 2: the intersection is trivial.

Our main result is a lower bound on the support of the generators of a space that has a certain degree of polynomial reproducibility. This problem was originally studied by Schoenberg for the single generated subspace $V=S(\phi)$ [23]. For an arbitrary number of generators however, the question has remained unsolved so far. In Theorem 1, we extend the result of Schoenberg to the case of two generators and propose a lower bound on $\left|\operatorname{Supp}\left(\phi_{1}\right)\right|+\left|\operatorname{Supp}\left(\phi_{2}\right)\right|$.

Theorem 1. Let $V=S\left(\phi_{1}, \phi_{2}\right) \subseteq L_{2}(\mathbb{R})$ be a shift-invariant with the ability to reproduce polynomials of degree up to $M$. Then, $\left|\operatorname{Supp}\left(\phi_{1}\right)\right|+\left|\operatorname{Supp}\left(\phi_{2}\right)\right| \geq M+1$.

Proof. We prove the theorem by contradiction. Assume that $V=S\left(\phi_{1}, \phi_{2}\right)$ can reproduce polynomials of degree up to $M$ and $\left|\operatorname{Supp}\left(\phi_{1}\right)\right|+\left|\operatorname{Supp}\left(\phi_{2}\right)\right|<M+1$. This implies that both $\phi_{1}$ and $\phi_{2}$ are indeed compactly supported functions. Denote their support by $\operatorname{Supp}\left(\phi_{1}\right)=\left[x_{1}, y_{1}\right]$ and $\operatorname{Supp}\left(\phi_{2}\right)=\left[x_{2}, y_{2}\right]$, respectively. We then consider the natural decompositions

$$
\left|\operatorname{Supp}\left(\phi_{i}\right)\right|=y_{i}-x_{i}=p_{i}+r_{i} \quad p_{i} \in \mathbb{Z}, r_{i} \in[0,1),
$$

for $i=1,2$. Now, define the intervals $I_{i, T}=\left[T+x_{i}+r_{i}, T+\right.$ $\left.x_{i}+1\right]$, and $J_{i, T}=\left[T+x_{i}, T+x_{i}+r_{i}\right]$ for $i=1,2$ and all $T \in \mathbb{Z}$. These are equidistant intervals of lengths $\left(1-r_{i}\right)$ and $r_{i}$, respectively.

We can readily check that, for any $a[\cdot] \in \ell_{2}(\mathbb{Z})$,

$$
\begin{aligned}
& \forall x \in I_{i, T}: \sum_{k \in \mathbb{Z}} a[k] \phi_{i}(x-k)=\sum_{k=T-p_{i}+1}^{T} a[k] \phi_{i}(x-k), \\
& \forall x \in J_{i, T}: \sum_{k \in \mathbb{Z}} a[k] \phi_{i}(x-k)=\sum_{k=T-p_{i}}^{T} a[k] \phi_{i}(x-k) .
\end{aligned}
$$

Finally, we define $I_{i}=\bigcup_{T \in \mathbb{Z}} I_{i, T}$ and $J_{i}=\bigcup_{T \in \mathbb{Z}} J_{i, T}$ for $i=1,2$. We now consider the problem in two cases (In Figure 1, we illustrate the cases visually):

Case 1: There exist $T_{1}, T_{2} \in \mathbb{Z}$ such that $U=I_{1, T_{1}} \cap I_{2, T_{2}}$ is a nontrivial interval.

Consider the space of polynomials of degree up to $M$ over the interval $U$ and denote it as $\Pi(U)$. On one hand, $\Pi(U)$ is an $(M+1)$-dimensional vector space spanned by the monomials $x^{m}$ for $m=0,1, \ldots, M$. On the other hand, from (11) and (13), we know that $\Pi(U)$ is spanned by the union of 

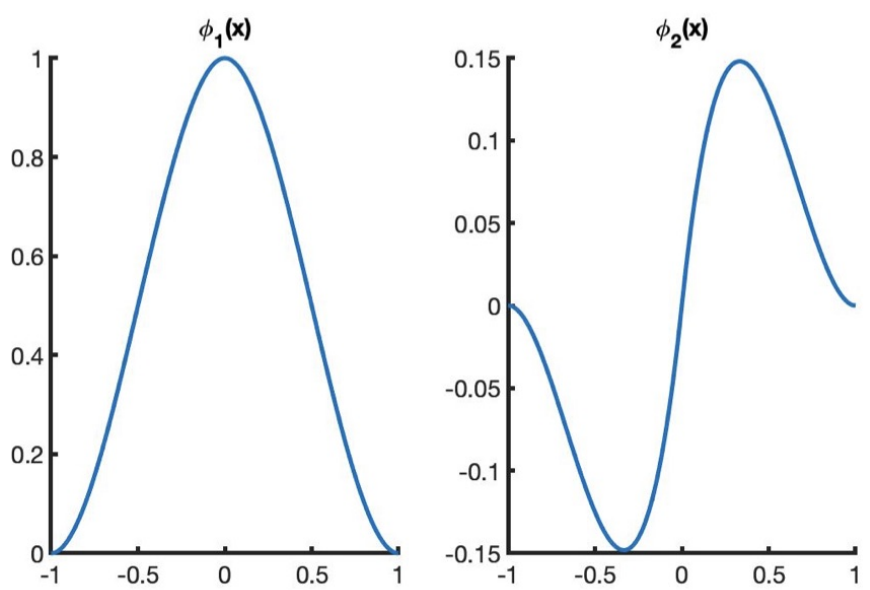

$$
S_{2}+S_{3}
$$

Fig. 2. Minimal-support generators for the space $S_{2}+S_{3}$ (Hermite splines).

$\left\{\phi_{1}(\cdot-k)\right\}_{k=T_{1}-p_{1}+1}^{T_{1}}$ and $\left\{\phi_{2}(\cdot-k)\right\}_{k=T_{2}-p_{2}+1}^{T_{2}}$. Therefore, the number of generators should be at least $M+1$. This yields that

$$
p_{1}+p_{2} \geq M+1 .
$$

Moreover, we have $\left|\operatorname{Supp}\left(\phi_{1}\right)\right|+\left|\operatorname{Supp}\left(\phi_{2}\right)\right| \geq p_{1}+p_{2}$ which together with (14), results in a contradiction with the original assumption.

Case 2: There are only countably many points in $I_{1} \cap I_{2}$ (the intersection is trivial). This implies that $\left|I_{1,0}\right|+\left|I_{2,0}\right| \leq 1$. Simplifying this inequality leads to

$$
r_{1}+r_{2} \geq 1 \text {. }
$$

Moreover, there exist $T_{1}, T_{2} \in \mathbb{Z}$ such that $U=I_{1, T_{1}} \cap J_{2, T_{2}}$ is a nontrivial interval. Similar to Case 1 , we consider $\Pi(U)$. Then, Eq. (11), and (13) imply that $\Pi(U)$ is spanned by $p_{1}+$ $\left(p_{2}+1\right)$ functions. Hence,

$$
p_{1}+p_{2}+1 \geq M+1 .
$$

Together with (15), we finally obtain that

$$
\left|\operatorname{Supp}\left(\phi_{1}\right)\right|+\left|\operatorname{Supp}\left(\phi_{2}\right)\right| \geq M+1 \text {. }
$$

\section{DISCUSSION}

In this section, we discuss our theorem by providing some examples. We consider spline spaces; they have been shown to be desirable from both theoretical and practical aspects [17], [24].

A spline of degree $n$ is a piecewise polynomial function of degree $n$ that has $(n-1)$ continuous derivative [23]. It is called a cardinal spline if the junctions (known as knots) are located on the integer grid. The space of cardinal splines of degree $n$ is denoted by $S_{n}$.

The spline space $S_{n}$ has the minimal-support generator $\beta^{n}$ : the B-spline of degree $n$. Its support $(n+1)$ is known to be the smallest among all cardinal splines [23]. Along with other

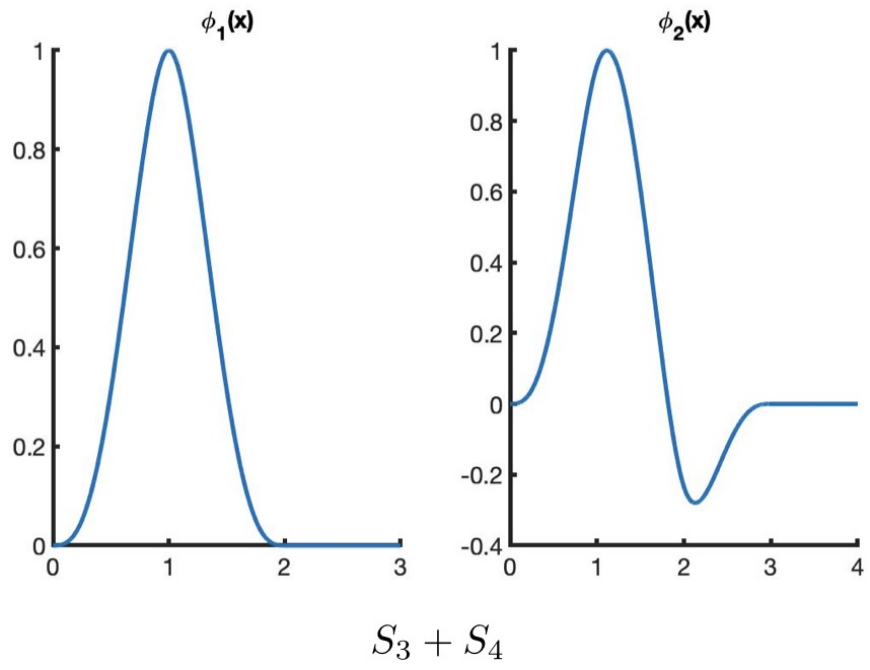

Fig. 3. Minimal-support generators for the space $S_{3}+S_{4}$. The sum of supports is 5 . It is tight due to our theorem. The sum of the supports of the canonical generators $\beta_{3}$ and $\beta_{4}$ is 9 , which is nearly twice the optimal value.

properties, their short support makes them a powerful tool in signal processing and sampling [25], [26].

As mentioned earlier, a single cardinal spline space has not sufficiently many degrees of freedom for derivative sampling. It is then natural to consider the sum of two consecutive spline spaces $S_{n-1}+S_{n}$. We assume that $n \geq 3$ in order to have continuous derivatives, which is crucial for this application. Since $S_{n}$ reproduces polynomials of degree $n$, the same obviously holds true for $S_{n-1}+S_{n}$.

The canonical generators of $S_{n-1}+S_{n}$ are $\beta^{n-1}$ and $\beta^{n}$. While these B-splines are the minimal-support generators of $S_{n-1}$ and $S_{n}$ separately, their support is not optimal for generating the sum space $S_{n-1}+S_{n}$. Their sum of support is $n+(n+1)=2 n+1$, which is almost twice the lower bound $(n+1)$ obtained from Theorem 1 .

In Figure 2 and Figure 3, we plot the minimal-support generators for the sum of spline spaces $S_{2}+S_{3}$ and $S_{3}+S_{4}$, respectively. Note that the equality case of our bound happens in these examples. We conjecture that our bound is tight for the sum of spline spaces $S_{n-1}+S_{n}$ for all $n \geq 3$.

Finally, it is worth to mention that the case $S_{2}+S_{3}$ is of special importance. The corresponding pair of generators shown in Figure 2 are the unique interpolatory generators of $S_{2}+S_{3}$ (obtained from (10)). These generators are known as the Hermite splines [27]. Being interpolatory while having the smallest support makes them a powerful tool in derivative sampling and related applications such as curve parametrization [28].

\section{CONClusion}

We have considered derivative sampling over a shiftinvariant reconstruction space generated by two compactly supported functions. The space is assumed to reproduce polynomials up to a certain degree. We have showed that the polynomial reproducibility imposes a lower bound on the sum of supports of the generators. Then, we have illustrated the 
remarkable tightness of our bound in the sum of spline spaces. A generalization of our theory to higher-order derivatives and also the construction of the optimal spline generators are possible research directions.

\section{ACKNOWLEDGMENT}

This work was funded by the Swiss National Science Foundation under Grant 200020 184646 / 1.

\section{REFERENCES}

[1] A. Papoulis, "Generalized sampling expansion," IEEE Transactions on Circuits and Systems, vol. 24, no. 11, pp. 652-654, November 1977.

[2] C. E. Shannon, "Communication in the presence of noise," Proceedings of the IRE, vol. 37, no. 1, pp. 10-21, Januray 1949.

[3] A. J. Jerri, “The Shannon sampling theorem-its various extensions and applications: A tutorial review," Proceedings of the IEEE, vol. 65, no. 11, pp. 1565-1596, November 1977.

[4] M. Unser, "Sampling-50 Years after Shannon," Proceedings of the IEEE, vol. 88, no. 4, pp. 569-587, April 2000.

[5] J. Kybic, T. Blu, and M. Unser, "Generalized sampling: A variational approach-Part I: Theory," IEEE Transactions on Signal Processing, vol. 50, no. 8, pp. 1965-1976, August 2002.

[6] —_ "Generalized sampling: A variational approachPart II: Applications," IEEE Transactions on Signal Processing, vol. 50, no. 8, pp. 1977-1985, August 2002.

[7] J. M. Medina and B. Cernuschi-Frías, "On the Papoulis sampling theorem: Some general conditions," IEEE Transactions on Information Theory, vol. 64, no. 10, pp. 6722-6730, October 2018.

[8] K. Gröchenig, J. L. Romero, and J. Stöckler, "Sampling theorems for shift-invariant spaces, Gabor frames, and totally positive functions," Inventiones mathematicae, vol. 211, no. 3, pp. 1119-1148, March 2018.

[9] L. Fogel, "A note on the sampling theorem," IRE Transactions on Information Theory, vol. 1, no. 1, pp. 47-48, March 1955.

[10] D. Linden, "A discussion of sampling theorems," Proceedings of the IRE, vol. 47, no. 7, pp. 1219-1226, July 1959.

[11] C.-C. Tseng and S.-L. Lee, "Design of wideband fractional delay filters using derivative sampling method," IEEE Transactions on Circuits and Systems I: Regular Papers, vol. 57, no. 8, pp. 2087-2098, August 2010.

[12] D. M. Santos and P. J. S. Ferreira, "Reconstruction from missing function and derivative samples and oversampled filter banks," in Proceedings of the IEEE International Conference on Acoustics, Speech, and Signal Processing (ICASSP'04), vol. 3, Montreal, Que., Canada, 17-21 May, 2004, pp. 941-944.

[13] M. Unser and J. Zerubia, "A generalized sampling theory without band-limiting constraints," IEEE Transactions on Circuits and Systems-II: Analog and Digital Signal Processing, vol. 45, no. 8, pp. 959-969, August 1998.

[14] —, "Generalized sampling: Stability and performance analysis," IEEE Transactions on Signal Processing, vol. 45, no. 12, pp. 2941-2950, December 1997.

[15] C. Deboor, R. A. DeVore, and A. Ron, "The structure of finitely generated shift-invariant spaces in $L_{2}\left(\mathbb{R}^{d}\right)$," Journal of Functional Analysis, vol. 119, no. 1, pp. 3778, Januray 1994.

[16] C. De Boor, R. A. DeVore, and A. Ron, "Approximation from shift-invariant subspaces of $L_{2}\left(\mathbb{R}^{d}\right)$," Transactions of the American Mathematical Society, vol. 341, no. 2, pp. 787-806, 1994.

[17] M. Unser, "Splines: A perfect fit for signal and image processing," IEEE Signal Processing Magazine, vol. 16, no. 6, pp. 22-38, November 1999.

[18] S. G. Mallat, "A theory for multiresolution signal decomposition: The wavelet representation," IEEE Transactions on Pattern Analysis and Machine Intelligence, vol. 11, no. 7, pp. 674-693, July 1989.

[19] M. Vetterli and J. Kovačević, Wavelets and Subband Coding. Prentice Hall Englewood Cliffs, 1995, vol. 995.

[20] G. Strang and G. Fix, "A Fourier analysis of the finite element variational method," in Constructive Aspects of Functional Analysis. Springer, 2011, pp. 793-840.

[21] A. Aldroubi and M. Unser, "Sampling procedures in function spaces and asymptotic equivalence with Shannon's sampling theory," Numerical Functional Analysis and Optimization, vol. 15, no. 1-2, pp. 1-21, 1994.

[22] W. Rudin, Functional Analysis, ser. International series in pure and applied mathematics. McGraw-Hill, 2006.

[23] I. J. Schoenberg, Cardinal Spline Interpolation. SIAM, 1973, vol. 12.

[24] C. De Boor, A Practical Guide to Splines. SpringerVerlag New York, 1978, vol. 27.

[25] — , "On calculating with B-splines," Journal of Approximation Theory, vol. 6, no. 1, pp. 50-62, 1972.

[26] M. Unser, A. Aldroubi, and M. Eden, "B-Spline signal processing: Part I-Theory," IEEE Transactions on Signal Processing, vol. 41, no. 2, pp. 821-833, February 1993.

[27] P. R. Lipow and I. Schoenberg, "Cardinal interpolation and spline functions. III. Cardinal Hermite interpolation," Linear Algebra and its Applications, vol. 6, pp. 273-304, Januray 1973.

[28] V. Uhlmann, J. Fageot, and M. Unser, "Hermite snakes with control of tangents," IEEE Transactions on Image Processing, vol. 25, no. 6, pp. 2803-2816, June 2016. 